\title{
Modeling Mobility Pattern for the Corona-Virus Epidemic Spread Propagation and Death Rate in Nigeria using the Movement-Interaction-Return Model
}

\author{
Arnold Adimabua Ojugo ${ }^{1}$, Obinna Nwankwo ${ }^{2}$ \\ ${ }^{1}$ Department of Computer Science, Federal University of Petroleum Resources Effurun, ojugo.arnold@ fupre.edu.ng, \\ ${ }^{2}$ Department of Computer Science, Novena University Ogume, tuk2obinna@ gmail.com
}

\begin{abstract}
Modeling the propagation rate of diseases in a society via social interaction has continued to pose its many challenges. The recent spread of the covid-19 epidemic cannot be left out. Thus, social interactions heralds its many benefits and has becomes a vehicle for epidemic outbreaks - which has continually left the world puzzled as the disease itself has come to stay. The nature of its rapid propagation on exposure alongside its migration spread pattern of this contagion (with retrospect of other epidemics) on daily basis, has also left experts rethinking the set protocols. Our study models the spread propagation of the corona-virus contagion using the movement-interaction-return on a social graph. Thus, we seek to measure if the corona-virus (covid-19) spread propagation can be minimized alongside its death rate using movement pattern as a threshold feature and set of protocols. We design a Markovian block model to help minimize targeted propagation with the advent of seed-node(s) using the susceptible-infect structure on a time-varying graph. Study results showed that movement pattern must be employed as an imperative factors when modeling the propagation of contagion(s).
\end{abstract}

Key words: contagions, movement-interaction-return MIR, propagation, agent-based model, decision support, migration.

\section{INTRODUCTION}

Overtime, mathematical models have been translated using computers as veritable tools to simulate and predict future states in complex, stochastic and dynamic phenomenon. These tools have thus, become useful, critical and imperative in controlling existing states of known events as well as helpful in prediction (as insight) to unknown events so long the proper and effective parameters are employed and adequately represented within the mathematical model $[1,2]$. These have been used to control/manage existing contagions epidemics as well as for analyzing cum forecasting the outcome therein of such epidemics $[3,4,5]$.
Diseases are disorders that occurs within the structure and function of any human, plant or animal - producing a specific (set of) symptoms. At its primitive stage, diseases exists in four dimensions namely thus: infectious, deficiency, hereditary, and non-hereditary. Diseases can also be viewed as harmful deviation(s) from the normal functioning of a system as it exhibit symptoms indicative of an anomaly, or its abnormal state $[6,7]$. Diseases are propagated from a system to another via a medium [8] as contagion to result in regular spread and epidemic - as it impacts on the society $[9,10,11]$. Many such tasks are modeled via a set of interconnected nodes on a social graph with their corresponding relations, as edges that connects the various nodes [7].

With mobility pattern of these nodes (also referred to as actors or agents), traces of the contagion can begin to emerge as a pattern as these actors migrate, interact and return to the point of their origin. These in turn, continues to impact on the changes and transformation process that often escalates minute and local disease outbreaks - morphing unto epidemics on a global scale $[12,13]$ and in turn - continued to necessitate the modeling of mobility pattern of actors alongside their varied interactions as integral facet of epidemic models - as these will help to effectively design and simulate such outbreak episodes as well as to help implement efficiently, a worthy as well as an efficient immunization cum preventive policies $[14,15,16]$.

\section{LITERATURE REVIEW}

Migration and interaction processes from persons living in a society - have continued to advance globalization. Its demerit also - has been in its acceleration of epidemic processes and its geographic expansion that multiplies and escalates local outbreaks into global epidemics. These factors have also been driven by frontier features namely: (a) migration activities from one place to another, and (b) effects of climate change [5, 13]. As a direct consequence of these two factors above, we have assisted in the spread of the Ebola outbreak as well as the current corona virus (covid-19) [3, 10]. Thus, it has become imperative that the inclusion of actor migration and interaction activities in the well-formulation of an epidemic propagation model has become mandatory as well as critical to achieving accurate description of any epidemic scenario [17]. Many algorithms and models have 
been posited leveraging on graph theories as their backdrop as means to address these growing and ever-increasing concerns [8].

\subsection{The Dynamic Susceptible-Infect (SI) Model}

As diffusion process continues at $\mathrm{t} \geq 0$, actors are first exposed (as seed-set nodes) to allow them form their perception and behaviour about the innovation. In time, as more of the actors are exposed to innovation, they make preferences - and based on their threshold (behaviour), adopt the innovation and form clusters and cliques via learning outcome. These strengthen their ties as well as improves the actor's personal network and consequently, helps them better retain information within their memory in time as the system continues in its search for optimality $[17,18,19,20,21]$. The random exchange in an actor's personal network allows knowledge swap - so as to yield in time agents with a new set of disposition (consider adoption and behaviour change cum evolution). As more adoption is encountered, more agents continue to learn/retain contents within their memories that better their personal network via community-influences. The listing in Algorithm 2 shows the SI (susceptible-infect) model [22, 23, 24, 25]:

Listing of the SI Algorithm

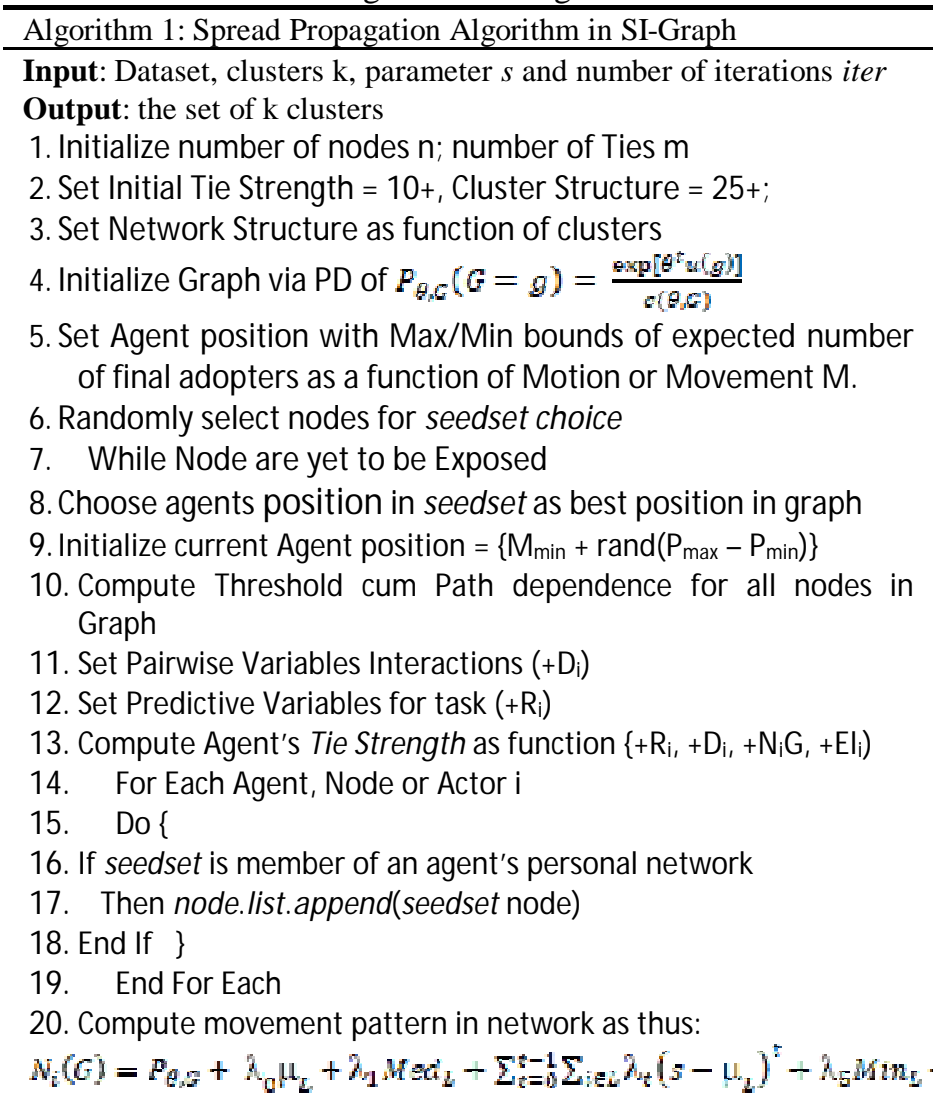

21. Compute actors change in behaviour from exposure at $t \geq 0$ as: $M_{\text {new }}=\mathrm{W} * \mathrm{M}_{\text {old }}+\mathrm{C}_{1} *$ rand ()$*\left[\left(\mathrm{P}_{\mathrm{i}}\right) / \mathrm{T}\right]+\mathrm{C}_{2} *$ rand ()$\left.*\left[\left(\mathrm{P}_{\mathrm{n}}\right) / \mathrm{T}\right)\right]$ 22. Updating agents' positions as $P_{\text {new }}=\left(P_{\text {old }}-M_{\text {new }}\right)$

23. // continue till all nodes are exposed, implies stop criterion reached
Each exposure yields an updated number of final adopters as its optimal solution in time via recomputed threshold value for exposed actors. We note that: (a) the agent position range is normalized between [0-1] dividing it by maximum range of agents, (b) each position randomly determines swap type needed for adoption rate, and (c) positions are reset and these recomputed new values will eventually reflect system threshold. With each solution found, model restarts with another randomly selected point for the planted seed-set choice in the graph space [26, 27].

Agents with threshold value above 0.5 are chosen. Process continues till all agents are exposed time $\geq \mathrm{t}-1$ at which all agents will have a threshold of 1 for the diffusion process or the nodes are continuously re-evaluated till an agent is found of threshold lesser than or equal to start-off threshold value (these form the stopping criterion for the model). At which point the solution is reached [28].

\subsection{Traditional Markovian Model}

The Markov Model is a double embedded chain that seeks to model complex chaotic processes as a chain of state sequences with probabilities associated to transition between states. For an $n$-order Markov, its transition probabilities depend on current and n-1 previous states, and used to determine states generated for each state observation in a series. A variant of the Markov model is the Hidden Markov and Profile Hidden Markov - both of which seeks to address the fundamental issues of Markov by: (a) makes explicit use of positional (alignment) data contained in sequences, and (b) it allows null transitions, where necessary so that the model can match sequences that includes insertion and deletions [29].

With state transition and probability distribution on nodal state observations, the Markovian Equation can be expressed as $\lambda=(\mathrm{A}, \mathrm{B}, \pi)$ depending on task [30]. Markovian models are best suited for tasks [31-32] for which (not limited to) these following conditions hold(s):

a. Problem 1: Given observations and parameters N and M, determine model $\lambda=(\mathrm{A}, \mathrm{B}, \pi)$ that best fits sequence. We train model to fit data. HMM training requires no aprior assumptions about the model other than outline parameter $\mathrm{N}$ and $\mathrm{M}$, which specifies the size of the model.

b. Problem 2: Compute probability that the given model produces an observation sequence if given the model $\lambda-$ $(\mathrm{A}, \mathrm{B}, \pi)$ and an observation sequence $\mathrm{O}$, compute $\mathrm{P}(\mathrm{O} / \lambda)$.

c. Problem 3: Uncover HMM $\lambda=(A, B, \pi)$ and observation sequence $\mathrm{O}$ to determine most likely sequence of states $\mathrm{X}$ $=\left(\mathrm{x}_{1}, \mathrm{x}_{2}, \ldots, \mathrm{x}_{\mathrm{T}}\right)$ that could have produced the sequence.

\subsection{Markovian Movement-Interaction-Return Ensemble}

Soriano-Panos et al [31] proposed an extended Markovian movement-interaction-return model as seen with the figure 1 . It first, notes that every time-step consists of three (3) stages namely:

a. Stage 1: Movement: Here, actors move across social graph with a probability $p$ - and each subgraph can be populated 
with actors from different geographical regions - so that the graph is etched with behavioral feats accompanying visitors and residents in a small-world graph as they possess very different migration pattern. Thus graph, introduces an object or parameter to govern migration flow - more complex than Origin-Destination matrices termed $M_{j}^{i}$ - a 3-D tensor that describes the probability with which an actor $i$ moves from subgraph $j$ to subgraph $k$ as in Eq, 1.

$$
M_{j}^{i} k=\delta_{j}^{i} R_{k}^{j}+\left(1+\delta_{j}^{l}\right) \delta_{k}^{j}
$$

This parameter imposes and ensures only actors located at their residence are allowed to move to other neighborhood areas. Thus, visitors staying out of their residence are forced to stay at their temporal node until coming back home. Once these movements takes place - actors initiate interaction and then learning can occur.

b. Stage 2: Interaction: With the SI-Susceptible model for disease propagation, social graph assumes that all actors are well-mixed as actors interact with those in their immediate neighborhood. Thus, an actor/node $x$ has several chances of exposing itself to another node $y$ and has exactly one chance of infecting the node $y$. Thus, the probability that an infected actor $x$ transmits the innovation (in this case, the disease pathogen) to a susceptible node actor $\mathrm{y}$ is given by $\lambda$; while, the probability that an infected actor overcomes the disease (that is, the disease dies out and the node is unable to propagate and not become susceptible again) is $\mu$.

c. Stage 3: Return: Here, visitors may decide to stay off their residence or to come back home with a probability of $\gamma^{-1}$.

This feature and parameter accounts for permanence time in destination node. Note, that $\gamma^{-1}-1(\gamma-1)$ recovers the solution to the original MIR model. Consequently, the propagation $\left(\gamma^{-1}=0\right)$ allows the actor to lose data about their residence and thus, are steadily redistributed across the neighboring areas.

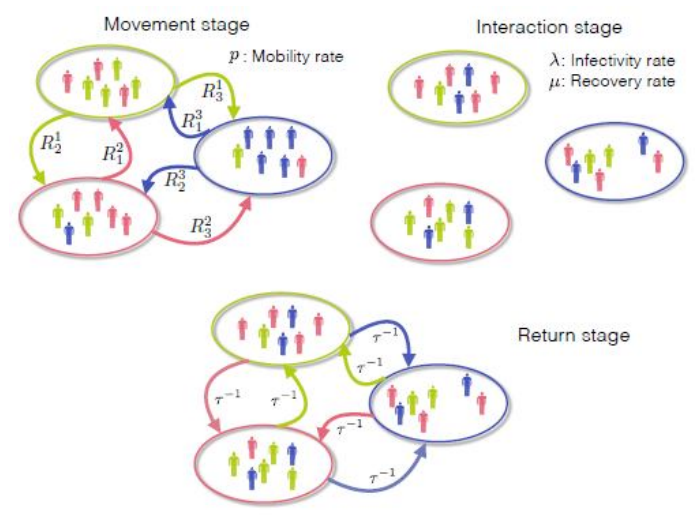

Figure 1. Movement-Interaction-Return Schematic diagram
The experiment seeks to explore the propagation death rate of the covid-19 epidemic as it ripple effects across a social graph using mobility as a predictor and threshold parameter for nodal adoption or rejection of the covid-19, as an innovation for diffusion or propagation. Thus, we seek to find the expected number of final adopters via network parameters with the actors' position in a social graph as they locally interact over-time. The study is more interested in the local emergent feats emanating from large-scale effects of such interacting actors of the entire supply network.

\section{FINDINGS / DISCUSSION}

\subsection{Data Gathering}

There are over eight isolation centers per senatorial district as created by the Delta State Government in response to curb the spread of the covid-19 pandemic. Figure 2 shows the epidemic curve for February 27, 2020 to April 23, 2021 of the NCDC daily epidemic curve in Nigeria with known reported cases of the pandemic in Delta State as given by table 1 [34].

Table 1. Nigeria Covid-19 Fact-sheet for April 232021

\begin{tabular}{|l|l|l|}
\hline No & Description of Cases & Population \\
\hline \multirow{3}{*}{1} & Total Samples Tested & 1868 \\
& Previously Confirmed Cases & 569 \\
& Newly Confirmed Case(s) & 37 \\
\hline 2 & Previously Discharged Cases & 136 \\
& Newly Discharged Cases & 6 \\
\hline 3 & Total Deaths & 20 \\
\hline 4 & Total Active Cases & 413 \\
\hline 5 & Total Number of Patients Abscond & 0 \\
\hline 6 & Days since last reported case & 0 \\
\hline
\end{tabular}

With the consequent spread of the Corona-virus (covid-19) in Nigeria alongside the migration of patients and individuals within and outside of the various states within the nation and abroad, it called on the urgency by the Federal Government of Nigeria to declare travel cum migration bans both intra-states, inter-states and outside of the Nigeria state.

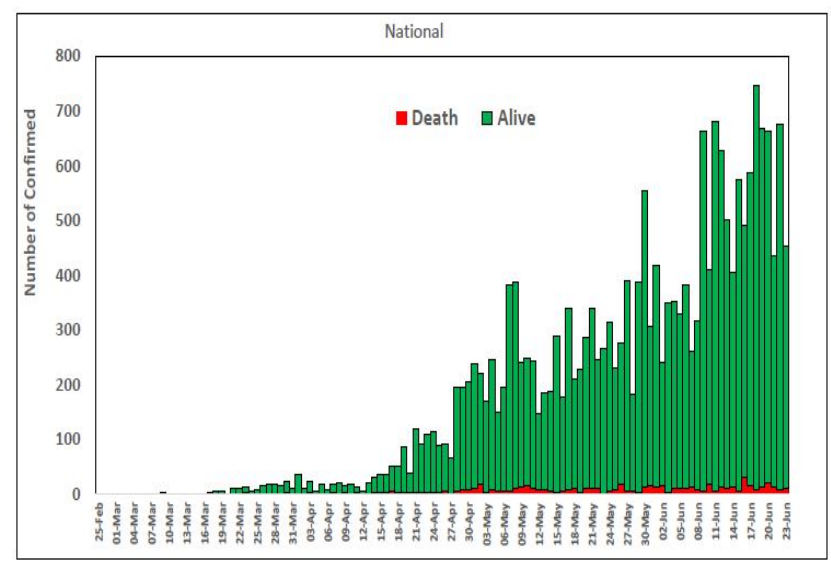

Figure 2. Confirmed cases (Feb 27 - April 23, 2021) 


\subsection{Data Pre-Processing and Verification}

Machine learning algorithm often requires gathering of the necessary dataset as cases, examples, and instances of all possible object classes. This is because in order to effectively train the adopted and adapted model, instances of the dataset must be appropriately labeled in order to minimize the error rate in the classification. These errors discern cum determine how effective and efficient the model progressed and is able to mine the data features of interest. Also, these errors can be resultant of the fact that in grouping cum labeling the data, some data points - even when not in the same class or group, can have lots of similarities under the unsupervised learning, or as they are used to predict new objects in a class under supervised learning. Also, the dataset must also be formatted appropriately to be used by the model; Else, it will result in data-type mismatch as the users tries to encode the data (via pre-processing stages) so that model is adequately trained to classify the data points into their corresponding classes [35].

For this study, we retrieved the data from Federal Medical Centre Epidemiology laboratory (Epi-lab) in Asaba, Delta State in Nigeria. The dataset contains about 54 attributes and 4,687 instances, including personal data of the patient, symptoms the patient suffers from, HIV and other tests, history of the disease, diagnostic tools used, treatment that includes regimens for the type of the disease and doses given, with its drug reaction, the follow-up results for the whole treatment period, also costs and hospitalization paid. However, the attributes that are likely to affect the patient behavior towards the treatment (treatment outcome is one of the following: cured, treatment completed, treatment failed, treatment discontinued, death, and transferred out). The dataset was further categorized into: (a) Attributes related to particular patient (age, sex, etc), (b) Attributes that are related to regimen, (c) Attributes that are related to proximity to health center, (d) Attribute related to treatment's side effect (social or clinical) and (e) attributes related to duration of treatment.

\section{FINDINGS / DISCUSSION}

\subsection{Findings and Discussion}

The application of MIR model with 5-fold training and test in evaluating the prediction model based on the correctly classified instances, the model has produced 93.7563 percent accuracy rate (see Table 2 ) showing confusion matrix with the five classes $(a, b, c, d, e)$ representing the various treatment outcome groups. A confusion matrix represents per true and false classes correct classification. Table 2 shows confirmed class of 568-cases. The discharged class of 134 cases correctly classified as true as in class (a); while, 2 others (from b-to-e) classified as false. Classes (a) and (b) respectively shows no significant difference between them. Thus, the error in the classification do not have significant effect. But, the general percentage obtained from software (correctly classified instances) for proposed Bayesian model is 93.7563 percent.

The study further shows that model adopts a graph-based approach where with the root represents the treatment outcome attributes; while the independent leaves represents other feats and attributes. Some of the assumption(s) made here includes that: (a) the variables are statistically independent, and (b) all variables are completely independent in accordance with [36] - though, it is observed that the independent assumption can barely arise [37, 38].

Table 2. Confusion Matrix and Percentage Achieved by Model

\begin{tabular}{|l|c|c|c|c|c|}
\hline \multicolumn{1}{|c|}{ Class } & $\mathrm{a}$ & $\mathrm{b}$ & $\mathrm{c}$ & $\mathrm{d}$ & $\mathrm{e}$ \\
\hline $\mathrm{a}=$ Confirmed & 568 & 1 & 0 & 0 & 0 \\
\hline $\begin{array}{l}\mathrm{b}=\text { Discharge } \\
\mathrm{d}\end{array}$ & 134 & 0 & 1 & 0 & 1 \\
\hline $\mathrm{c}=$ Death & 20 & 0 & 3 & 5 & 0 \\
\hline $\mathrm{d}=$ Active & 409 & 0 & 2 & 1 & 2 \\
\hline e=Absconded & 2 & 0 & 0 & 1 & 0 \\
\hline
\end{tabular}

Table 3 shows there is a relation between variables and its corresponding probabilities. The discharge class is dependent upon the variable (piw) by 0.962 probabilities. Thus, we can violate the independent assumption that: "no more than one parent". This is expressed by the set (maxNrOfParents=1) with which this process leads to achieve 94-percent accuracy. We notice the relations between attributes and their affection to the prediction result in accordance with [28, 29].

Table 3. Probability Distribution of (piw) Variable

\begin{tabular}{|l|l|l|}
\hline Class & Yes & No \\
\hline a=Confirmed & 0.999 & 0.001 \\
\hline $\begin{array}{l}\text { b=Discharge } \\
\text { d }\end{array}$ & 0.962 & 0.038 \\
\hline c=Death & 0.107 & 0.893 \\
\hline d=Active & 0.01 & 0.99 \\
\hline e=Absconded & 0.136 & 0.864 \\
\hline
\end{tabular}

\subsection{Result Trade-offs}

Several trade-off were noticed during result compilation and they fall under these [39, 40, 41, 42, 43, 44]:

a. Result Presentation - researchers often display flawed results, modify and/or build new models rather than re-test limitations, biasness and inabilities of existing ones. Also, some researchers fail to report negative results thinking they are less valuable. We employ such data driven model to curb the non-linearity and dynamism in observed datasets used to train and test model, unlike knowledge models.

b. Efficiency - modelers use figure to show how well their simulations are, in agreement with observations (even with their limited data that is squeezed) with lines for observed and simulated results that are not easily distinguishable. Some researchers do not even provide numerical dataset used for their study; but their model is in 'good agreement' with observations. Some measure of goodness does not provide the relevant information and knowledge for the task at hand.

c. Insufficient Testing - Validation is a comparison of computed versus observed values, and many studies suffer from inadequate data. If a model/algorithm seeks to simulate results of a task, such capability cannot be 
demonstrated with unfounded/misleading result from limited data and misleading conclusions.

d. Model validation is a scientific dialogue - impeded by improper applications and ambiguous results.

\section{CONCLUSION}

In the study, we employed an MIR framework as predictive techniques to construct a target model for predicting potential spread and classification of the covid-19 contagion. Moreover, this study used medical dataset gathered from Federal Medical Center Epidemiology laboratory in Delta State. Model yields an acceptable accuracy with improved performance via the violation of the independent assumptions. This violation was based on the probability distribution of attributes. The model will serve as useful and cost-effective tool in a health care system wherein there exist limited resources. Furthermore, the model can be used for modeling predictive frameworks with migration cum mobility pattern therein to solve problems in other sphere. Finally, the authors of this study believed that the machine learning techniques used in this paper warrant further investigation, particularly to explore conditions and attributes under which this study was carried out as well as seek other conditions, in this underlined problem where they are most likely to be effective.

\section{REFERENCES}

1. World Health Organization. Coronavirus Disease (COVID-2019) Situation Reports. Available online: https://www.who.int/emergencies/diseases/novel-corona virus-2019/situation-reports (accessed on 2March 2020).

2. A.A. Ojugo., O.D. Otakore., Forging an optimized Bayesian network model with selected parameter for detection of Coronavirus in Delta State of Nigeria, Journal of Applied Science, Engineering, Technology. \& Education, 3(1): pp37-45, doi: 10.35877/454RI.asci2163, 2020

3. A.A. Ojugo., A.O. Eboka., Modeling behavioral evolution as social predictor for coronavirus contagion and immunization in Nigeria, Journal of Applied Science, Engineering, Technology and Education, 3(2): pp37-45, doi: 10.35877/454RI.asci130, 2012.

4. O. Adegboye, A. Adekunle, A. Pak, E. Gayawan, D. Leung, D. Rojas, F. Elfaki, E. McBryde, D. Eisen, Change in outbreak and impact on importation risks of COVID-19 progression: modelling study. medRxiv 2020. [CrossRef]

5. M. Martinez-Alvarez, A. Jarde, E. Usuf, H. Brotherton, M. Bittaye, A.L. Samateh, M. Antonio, J. Vives-Tomas, U. D'Alessandro, A. Roca, COVID-19 pandemic in west Africa. Lancet Glob. Health, 2020. [CrossRef]

6. M. Gilbert, G. Pullano, G.; Pinotti, F.; Valdano, E.; Poletto, C.; Boëlle, P.-Y.; et al., Preparedness and vulnerability of African countries against importations of COVID-19: A modelling study. Lancet, 2020, 395, 871-877. [CrossRef]

7. A.A. Ojugo., E. Ekurume., Predictive intelligence decision support model in forecasting of diabetes pandemic using a reinforcement deep learning approach, International Journal of Education and Mgt. Engineering, 11(2), pp.40-48, doi: 10.5815/ijeme.2021.02.05, 2021.

8. A.A. Ojugo., R.E. Yoro., Migration pattern as threshold feature in propagation of covid-19 epidemic using actor-based model on social graph, Journal of Information and Visualization, 2(2): pp93-105, doi: 10.35877/454RI.jinav379, 2021

9. O. Adegboye, A. Adekunle, E. Gayawan, Early transmission dynamics of novel COVID-19, Int. J. Environmental Research and Public Health, medRxiv 2020. [CrossRef]

10. W. McKibbin, R. Fernando, The Global Macroeconomic Impacts of COVID-19: Seven Scenarios. 2020, [web]: retrieved from https://researchgate.net

11. A.A. Ojugo., O. Nwankwo., Multi-agent Bayesian framework for parametric selection in the detection and diagnosis of Tuberculosis contagion in Nigeria, Journal of Information and Visualization, 2(2): pp69-76, doi: 10.35877/454RI.jinav375, 2021

12. Worldometers (2020). Global Statistics on COVID-19 Pandemic Outbreak. Retrieved from, https://worldometers.info/coronavirus

13. R. Addi, A. Benksim, M. Amine, M. Cherkaoui, Asymptomatic COVID-19 infection management: The key to stopping COVID-19. Journal of Clinical and Experimental Investigations, 11(3), 1-2, 2020

14. A.A. Ojugo., F. Aghware., R. Yoro., M. Yerokun., A. Eboka., C. Anujeonye., F. Efozia., Predict behavioral evolution on graph model, Advances in Networks, 3(2): pp8-21, 2015

15. M. Azarafza, M. Azarafza, H. Akgunc, Clustering method of spread pattern analysis of corona-virus (covid-19) infection in Iran, J. Applied Science, Engineering Tech. \& Education, 3(1) pp1-6, doi: 10.35877/454ARI.asci31109, 2020.

16. J. Hellewell, S. Abbott, A. Gimma, N.I. Bosse, C.I. Jarvis, T.W. Russell et al. Feasibility of controlling COVID-19 outbreaks by isolation of cases and contacts, 2020, doi: 10.1371/journal.pone.0072168

17. Y. Bai, L. Yao, L., Wei, T., Tian, F., Jin, D, Y., Chen, L. Wang, Presumed asymptomatic carrier transmission of COVID-19. JAMA. 2020.

18. A.A. Ojugo., D.A. Oyemade., Predicting diffusion dynamics of coronavirus in Nigeria through ties-strength threshold on a cascading SI-graph, Technology Report of Kansai University, TRKU-13-08-2020-10998, 62(8): pp4313-4323, 2020

19. D. Acemoglu, V.M. Carvalho, A. Ozdaglar, A. Tahbaz-Salehi, The network origins of aggregate fluctuations, Econometrica, 80(5), pp.1977-2016, 2012 
20. J. Kim, J. Kim, S.K. Lee, L.R. Tang, Effects of epidemic disease outbreaks on the financial performance of restaurants: Event study method approach. Journal of Hospitality and Tourism Management, 43, 32-41, 2020

21. H. Jung, M. Park, K. Hong, E. Hyun, The impact of an epidemic outbreak on consumer expenditures: An empirical assessment for MERS Korea. Sustainability, 8(454), 1-15. DOI:10.3390/su8050454, 2016

22. M. Granovetter, Threshold Models of Collective Behavior. American J. Sociology, 83(6), p1420-1443. doi:10.1086/226707, 1978

23. A.A. Ojugo, A.O., Eboka, Mitigating technical challenges via redesigning campus network for greater efficiency, scalability and robustness: a logical view, Int. J. Modern Edu. \& Comp. Sci., 6, pp29-45, doi: 10.5815/ijmecs.2020.06.03, 2020

24. A.A. Ojugo, I.P. Okobah., Prevalence rate of hepatitis-B virus infection in Niger Delta region of Nigeria using graph-based diffusion heuristic model, IJCAOnline International Journal of Computer Application, 179(39): pp27 -33, 2018

25. A. Sala, N. Cao, C. Wilson, R. Zablit, H. Zheng, B.Y. Zhao, Measurement calibrated graph models for social network experiments, IW3C2, ACM 978-1-60558-799-8/10/04, 2010

26. E. Gilbert, K. Karahalois, Predicting tie strengths with social media, Journal of computer and Human Interface, 15, p76-97, ACM 978-1-60558-246-7/09/04, 2009

27. M.S. Handcock, K.J. Gile, Modeling social networks from sampled data*, Annals of Applied Statistics, arXiv: math.PR/00000, 2009.

28. R. Toivonen, L. Kovanena, M. Kiveläa, J.K. Onnela, J. Saramäkia, K. Kaskia, A comparative study of social networks models: network evolution and nodal attributes models, Social Networks, 31, p240-254, doi:10.1016/j.socnet.2009.06.004, 2009.

29. A.A. Ojugo, E. Ben-Iwhiwhu, O. Kekeje., M. Yerokun., I. Iyawah., Malware propagation on social time varying networks: a comparative study of machine learning frameworks, Int. J. of Modern Education Comp. Sci., 6(8): pp25-33, doi: 10.5815/ijmecs.2014.08.04, 2014

30. A.A. Ojugo, O. Nwankwo, Spectral-cluster solution for credit-card fraud detection using genetic algorithm trained modular deep learning neural network, J. of Info. \& Visualization, 2(1): pp15-24, doi: 10.35877/454RI.jinav274, 2021

31. D. Soriano-Panos, G. Ghoshal, A. Arenas, J. Gomez-Gardenes, Impact of temporal scales and recurrent mobility patterns on the unfolding of epidemics, 2019, Preprint - ResearchGate, arXiv: 1909.12731v1 [physics,soc.ph]: pp1-15.

32. C.C. Aggarwal, C.K. Reddy, Data Clustering: Algorithms and Applications. Chapman and Hall, 2013.

33. S. Schnettler, A small world on feet of clay? A comparison of empirical small-world studies against best-practice criteria, Social Networks, 31(3), p179-189, doi:10.1016/j.socnet.2008.12.005. 2009

34. Nigeria Centre for Disease Control [NCDC] (2020). COVID-19 Pandemic Outbreak. Retrieved from, https://covid19.ncdc.gov.ng

35. A.A. Ojugo, O.D. Otakore, Forging an optimized Bayesian network model with selected parameter for detection of the Coronavirus in Delta State, J. Applied Sci., Eng., Tech. \& Edu., 3(1): pp37-45, doi: 10.35877/454RI.asci2163, 2021

36. A.A. Ojugo, R.E. Yoro, Forging deep learning neural network intrusion detection framework to curb distributed denial of service attack, Int. J. of Elect. \& Computer Engr., 11(2): pp1498-1509, doi: 10.11591/ijece.v11i2.pp1498-1509, 2021

37. C. Wilson, B. Boe, A. Sala, K.P. Puttaswamy, B.Y. Zhao, User interactions in social networks and their implications. In Proc. of EuroSys (April 2009).

38. J.P. Scott, Social network analysis: A Handbook, (2nd Ed). 2000. Thousand Oaks, CA: Sage Publications.

39. T.S. Smith, G.T. Stevens, The architecture of small networks: strong interaction and dynamic organization in small social systems, American Sociological Review, 64, p403-20, 1999

40. D. Krackhardt, The Strength of Strong Ties: Importance of Philos, In N. Nohria and R. Eccles., Networks and Organizations: Structure, Form and Action (p216-239), Harvard Biz School Press, 1990

41. A.A. Ojugo., A.O., Eboka., E.O. Okonta., R.E. Yoro., F.O. Aghware., Genetic algorithm rule-based intrusion detection system, $J$. of Emerging Trends in Computing Information System, Vol. 3, No. 8, pp.1182-1194, 2012

42. A.A. Ojugo., D.O. Otakore., Intelligent cluster connectionist recommender system using implicit graph friendship algorithm for social networks, Int. J. Artificial Intelligence, $9(3)$ : pp497 506, doi: 10.11591/ijai.v9.i3.pp497 506, 2020.

43. A.A. Ojugo., A.O. Eboka., An intelligent Bayesian network to improve performance and dependability analysis of a campus network, International Journal of Artificial Intelligence, 10(3), pp [web]: ijai.iaescore.com/index.php/IJAI/article/view/20923

44. A.A. Ojugo., D.A. Oyemade., Boyer Moore string-match framework for a hybrid short messaging service spam filtering technique, International Journal of Artificial Intelligence, 10(3), doi: 10.11591/ijai.v10.i3.pp519-527 